\title{
Converting Level Set Gradients to Shape Gradients
}

\author{
Siqi Chen ${ }^{1 \star}$, Guillaume Charpiat ${ }^{2}$, and Richard J. Radke ${ }^{1}$ \\ 1 Department of ECSE, Rensselaer Polytechnic Institute, Troy, NY, USA \\ chens@rpi.edu, rjradke@ecse.rpi.edu \\ 2 Pulsar Project, INRIA Sophia-Antipolis, France \\ Guillaume.Charpiat@sophia.inria.fr
}

\begin{abstract}
The level set representation of shapes is useful for shape evolution and is widely used for the minimization of energies with respect to shapes. Many algorithms consider energies depending explicitly on the signed distance function (SDF) associated with a shape, and differentiate these energies with respect to the SDF directly in order to make the level set representation evolve. This framework is known as the "variational level set method". We show that this gradient computation is actually mathematically incorrect, and can lead to undesirable performance in practice. Instead, we derive the expression of the gradient with respect to the shape, and show that it can be easily computed from the gradient of the energy with respect to the SDF. We discuss some problematic gradients from the literature, show how they can easily be fixed, and provide experimental comparisons illustrating the improvement.
\end{abstract}

\section{Introduction}

In recent years, much work on geometric active contour models, i.e. active contour models [1] implemented with the level set method [2], has been proposed to solve many computer vision problems [3]. Any planar closed curve $\Gamma$, i.e. any function $\Gamma: \mathbb{S}^{1} \rightarrow \Omega$ from the circle $\mathbb{S}^{1}$ to the image domain $\Omega \subset \mathbb{R}^{2}$, can be represented by the zero level set of a higher-dimensional embedding function $\phi: \Omega \rightarrow \mathbb{R}$. During curve evolution, instead of directly updating the contour $\Gamma$, one can then update its associated embedding function $\phi$, which is more practical for handling topological changes, like merging or splitting. The embedding function $\phi$ is usually the signed distance function (SDF) of $\Gamma$, i.e. the function that associates any point $x \in \Omega$ with the signed distance $\phi(x)= \pm d(x, \Gamma)$ from $x$ to $\Gamma$, with a minus sign if $x$ belongs to the interior of the region delimited by $\Gamma$.

Many computer vision problems involving shapes can be formulated as the minimization of a certain energy functional $E(T)$. Depending on the properties of the energy $E$, one is often reduced to performing gradient descents with respect

\footnotetext{
* This work is supported in part by CenSSIS, the NSF Center for Subsurface Sensing and Imaging Systems, under the award EEC-9986821.
} 
to the shape $\Gamma$, starting from an initialization $\Gamma_{0}$ and making $\Gamma$ evolve step by step in the opposite direction of the gradient of the energy:

$$
\left\{\begin{array}{l}
\Gamma(0)=\Gamma_{0} \\
\frac{\partial \Gamma(t)}{\partial t}=-\nabla_{\Gamma} E(\Gamma(t))
\end{array}\right.
$$

where the gradient $\nabla_{\Gamma} E(\Gamma)$ is defined from the derivative of $E$ with respect to $\Gamma$ and depends on the choice of an inner product (see Section 3.1 for a proper definition, or [4]). If one chooses to represent the contour $\Gamma(t)$ by an embedding function $\phi(t)$, then one is interested in the equation that governs the evolution of $\phi(t)$. Since by definition $\forall t, \forall x \in \Gamma(t), \quad \phi(t)(x)=0$, one obtains, by differentiation, $\frac{\partial \phi}{\partial t}+\frac{\partial \phi}{\partial x} \frac{\partial \Gamma}{\partial t}=0$, and thus:

$$
\left\{\begin{array}{l}
\phi(0)=\phi_{0} \quad\left(\text { e.g. }:=\operatorname{SDF}\left(\Gamma_{0}\right)\right) \\
\frac{\partial \phi}{\partial t}(x)=\left|\nabla_{x} \phi(t)\right|(x) V(t)(x) \quad \forall x \in \Gamma(t)=\phi(t)^{-1}(0)
\end{array}\right.
$$

where $\forall x \in \Gamma, V(t)(x)=\nabla_{\Gamma} E(\Gamma(t))(x) \cdot \mathbf{n}_{\Gamma(t)}(x)$ is the normal velocity field, i.e. the part of the shape gradient that is normal to the contour $\Gamma$. This so-called "level set equation" has to be properly defined for points which are not on $\Gamma$, e.g. by extending the velocity field $V$ to at least a narrow band around $\Gamma$.

However, for many applications, calculating the gradient using Eq.(1) directly is difficult and therefore an alternative derivation to obtain the level-set equation was proposed in [5], named the "variational level set method". The idea is that since there is a bijection between $\Gamma$ and its signed distance function $\phi$, an energy defined on shapes $E(\Gamma)$ can be rewritten as an energy $F(\phi)$ defined on their level set representations and vice versa. Subsequently, one might be interested in deriving the Euler-Lagrange equation that minimizes $F(\phi)$ directly:

$$
\frac{\partial \phi}{\partial t}=-\nabla_{\phi} F(\phi) \quad=: G(\phi)
$$

Note that this equation is a priori not related to Eq.(2). Since the introduction of the variational level set approach, much work has been carried out under this framework, e.g. [6-8], to name a few. However only very few functions in $L^{2}(\Omega \rightarrow \mathbb{R})$ are SDFs of a shape; for instance, they must satisfy the Eikonal Equation $\left|\nabla_{x} \phi\right|=1$ almost everywhere. Therefore, a new $\phi$ obtained from a discrete step of Eq.(3) will generally not itself be a valid SDF. Various authors $[5,9,10]$ showed that if the velocity field $G$ in Eq.(3) satisfies

$$
\nabla_{x} G \cdot \nabla_{x} \phi=0
$$

then the evolving level set $\phi$ will always remain a valid SDF for all time. However, a general level set gradient $G=-\nabla_{\phi} F(\phi)$ is unlikely to satisfy Eq.(4), and several approaches have been proposed to maintain the SDF property of $\phi$ [11]. 
The first approach is known as "velocity extension" [9, 10]. A narrow band around $\Gamma$ is first defined, and Eq.(4) is solved to extend $G$ with the "Fast Marching" method [10]. A second approach is known as "reinitialization" [12]. Here, Eq.(3) is used to update $\phi$, but since the newly obtained $\phi$ will drift away from a SDF, the evolution is occasionally stopped, and the SDF of the zero level set of $\phi$ is recomputed. A third approach was proposed by Li et al. [13], in which the deviation of $\left|\nabla_{x} \phi\right|$ from 1 is incorporated into the energy function $F(\phi)$.

However, previous works neglect one important issue. Although the desired energy $E(\Gamma)$ can be rewritten as $F(\phi)$, the meaning of the gradient $\nabla_{\phi} F(\phi)$ is fundamentally different from $\nabla_{\Gamma} E(\Gamma)$ because the computation $\nabla_{\phi} F(\phi)$ is performed without the constraint that the gradient should belong to the very particular subset of variations of $\phi$ that maintain its property of being a SDF. Thus the effect of the gradient $\nabla_{\phi} F(\phi)$ on the zero level $\Gamma$ may be completely different from $\nabla_{\Gamma} E(\Gamma)$ and entirely incorrect. Updating the level set function to maintain the SDF property (e.g. by recomputing it from its zero level) does not change the fact that the newly obtained $\Gamma$ associated with $\phi$ is wrong.

In this work, we show that with a simple "velocity projection" step, the level set gradient can be made to exactly match the true gradient of $E(\Gamma)$ with respect to $\Gamma$, which we call the "shape gradient". Therefore, with our approach, one can still take the derivative $\nabla_{\phi} F(\phi)$ of an energy $F(\phi)$ with respect to $\phi$, and transform it to obtain the correct shape gradient to deform $\phi$. To motivate the discussion, Fig.1 compares our result with the standard variational level set method, where $E(\Gamma)$ is defined as the $L^{2}$ distance from $\phi$ to the SDF $\phi_{T}$ corresponding to a target curve $\Gamma_{T}$, i.e. $F(\phi)=\left\|\phi-\phi_{T}\right\|_{L^{2}}^{2}$. We can see that using the standard (incorrect) level set gradient, the initial curve would shrink to a point, while with our corrected gradient, the initial curve is correctly drawn to $\Gamma_{T}$. For more detailed discussion, please refer to Section 4 .

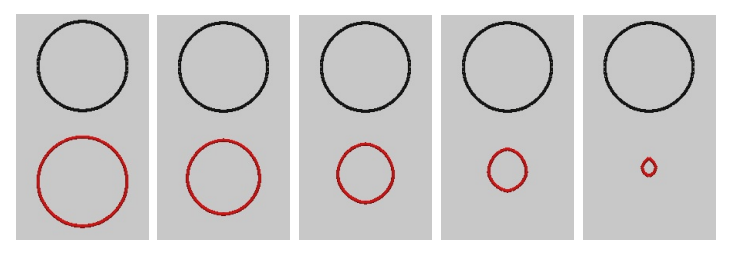

(a)

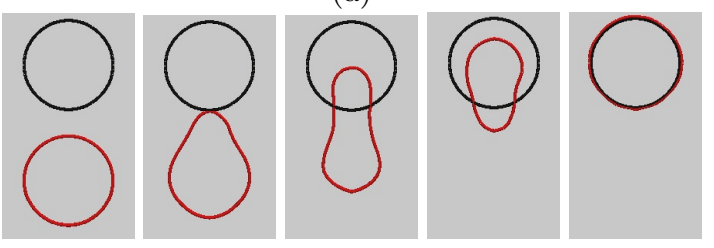

(b)

Fig. 1: Curve evolution of the red circle $\Gamma$, where the black circle $\Gamma_{T}$ is the target. The cost function is the $L^{2}$ distance between the two SDFs. (a) standard variational level set method, (b) our result. 
This paper is organized as follows. In Section 2, we describe the family of admissible SDF variations $\delta \phi$ so that $\phi+\delta \phi$ remains a valid SDF. In Section 3 , we use this family to draw the connection between the level set gradient obtained from Eq.(3) and the shape gradient from Eq.(1). We show that by projecting the level set gradient onto the family of admissible SDF variations, we exactly recover the shape gradient. Therefore, with only a simple "velocity projection" step, we can convert any level set gradient to the correct shape gradient and thus deform $\phi$ by extending the shape gradient with the usual velocity extension approach. We also show how to directly compute the correct deformation of $\phi$ by integrating the level set gradient over parts of the image $\Omega$, without explicitly computing the shape gradient. In Section 4, we review some often-used level set gradients from the literature that need the "velocity projection" step to make them correct deformations of $\Gamma$. We also show experimental results that compare the corrected shape gradient with problematic level set gradients. We then conclude and discuss future directions.

\section{The family of admissible SDF variations}

Let us consider a closed planar curve $\Gamma \in L^{2}\left(\mathbb{S}^{1} \rightarrow \Omega\right)$, an infinitesimal deformation field $\delta \Gamma \in L^{2}\left(\Gamma \rightarrow \mathbb{R}^{2}\right)$, which can be seen as a function in $L^{2}\left(\mathbb{S}^{1} \rightarrow \mathbb{R}^{2}\right)$ using $\Gamma$ 's parameterization, and let $\phi \in L^{2}(\Omega \rightarrow \mathbb{R})$ be the SDF associated with $\Gamma$. If we consider an infinitesimal variation $\delta \phi$ of $\phi$, which is any function in $L^{2}(\Omega \rightarrow \mathbb{R})$, in the general case $\phi+\delta \phi$ would not be a valid SDF of some corresponding shape. Thus we should only consider variations $\delta \phi$ so that there exists a shape $\Gamma^{\prime}$ so that $\phi+\delta \phi$ is the SDF of it, i.e. $\phi+\delta \phi=\Phi\left(\Gamma^{\prime}\right)$.

Let us call $\mathcal{F}$ the family of all such infinitesimal deformations $\delta \phi$. There is a bijection between $\mathrm{SDF}$ variations $\delta \phi$ in $\mathcal{F}$ and shape deformations $\delta \Gamma$ of $\Gamma$; that is, for any vector field $\delta \Gamma$ normal to $\Gamma$ at each point of $\Gamma$ (since tangent displacements do not affect the shape), we can associate a corresponding SDF variation $\delta \phi$ and vice versa. We show in the appendix that to match the shape deformation $\delta \Gamma$, one has to update $\delta \phi$ according to:

$$
\forall x \in \Omega, \quad \delta \phi(x)=-\delta \Gamma\left(s_{x}\right) \cdot \boldsymbol{n}_{\Gamma\left(s_{x}\right)}
$$

where $\Gamma\left(s_{x}\right)$ is the projection of point $x$ onto $\Gamma$, and $\boldsymbol{n}_{\Gamma\left(s_{x}\right)}$ is the unit normal at point $\Gamma\left(s_{x}\right)$ pointing outwards. Here $\delta \phi$ can be understood as $\frac{d \phi}{d \Gamma}(\delta \Gamma)$. Note however that $\frac{d \phi}{d \Gamma}$ is not defined when a topological change occurs, so that $\phi$ will have to be recomputed from its 0-level after topological changes.

Fig. 2 illustrates the admissible variations of an SDF. Intuitively, Eq.(5) implies that a valid deformation $\delta \phi$ at any point $x$ in $\Omega$ depends only on its projection onto $\Gamma$ : if two points share the same projection point $\Gamma\left(s_{x}\right)$, then their variation will be the same. This is a known result $[5,9,10]$. Consequently, all points on a projection line vary the same way, i.e. $\delta \phi$ is a constant along projection lines to $\Gamma$. Conversely, if $\delta \phi$ is constant along all projection lines to $\Gamma$, then there exists a deformation $\delta \Gamma$ associated with it. Note that the projection 


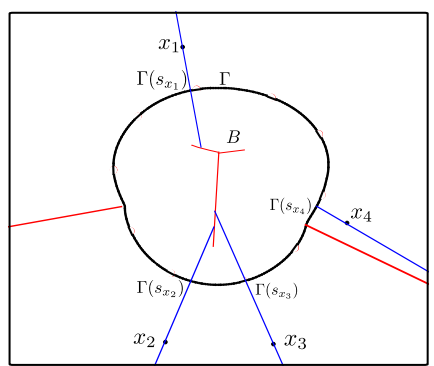

(a)

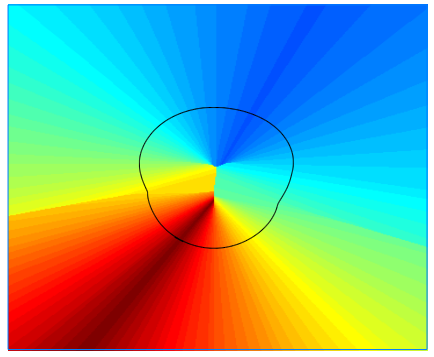

(b)

Fig. 2: Illustration of the admissible variations of a SDF. (a) The projection line (in blue) is the line going through $x$ that orthogonally intersects $\Gamma$ at $\Gamma\left(s_{x}\right)$ and stops at the skeleton $B$. (b) The admissible variation $\delta \phi$ is a constant along the projection lines.

$\Gamma\left(s_{x}\right)$ is well-defined for all points in $\Omega$ except for those on the skeleton of $\Gamma$. Since we will later integrate bounded variations over regions of $\Omega$ in which the Lebesgue measure of the skeleton is 0 , this will pose no problem in our work.

As a consequence, the family $\mathcal{F}$ of all admissible variations of a $\operatorname{SDF} \phi$ is the set of all $L^{2}(\Omega \rightarrow \mathbb{R})$ functions that are constant along projection lines to $\Gamma$. This means that, when performing a shape evolution based on a level set representation, one should ensure that the level set variation belongs to this family $\mathcal{F}$. Numerical algorithms such as the Fast Marching method [10] can be used to obtain such a level set variation based on the deformation of $\Gamma$.

\section{Velocity projection}

In the previous section, we defined the family of all admissible variations of an SDF as the set of all functions that are constant along projection lines to $\Gamma$. We will now use this result to draw a connection between $\nabla_{\phi} F(\phi)$, which we call the "level set gradient" and $\nabla_{\Gamma} E(\Gamma)$, which we call the "shape gradient". We will show that projecting $\nabla_{\phi} F(\phi)$ onto the family $\mathcal{F}$ will exactly produce $\nabla_{\Gamma} E(\Gamma)$.

\subsection{Gradients and inner products}

The gradient definition depends on the choice of the inner product in the tangent space of shapes $[14,15]$. In this work, we use the standard $L^{2}$ inner product:

$$
\left\langle\delta \Gamma_{1} \mid \delta \Gamma_{2}\right\rangle_{L^{2}\left(\mathbb{S}^{1} \rightarrow \mathbb{R}\right)}=\int_{\Gamma} \delta \Gamma_{1}(s) \cdot \delta \Gamma_{2}(s) d \Gamma(s)
$$

where $\delta \Gamma_{1}$ and $\delta \Gamma_{2}$ are two deformations of $\Gamma$, where $s$ denotes a parameterization of $\Gamma$, and where $d \Gamma(s)=\left\|\frac{d \Gamma}{d s}\right\|_{\mathbb{R}^{2}} d s$ is the associated differential element (i.e. its parameterization-independent length). The gradient associated with this inner product is then defined as the unique deformation $\nabla_{\Gamma} E(\Gamma)$ that satisfies

$$
\forall \delta \Gamma, \quad D E(\Gamma)(\delta \Gamma)=\left\langle\nabla_{\Gamma} E(\Gamma) \mid \delta \Gamma\right\rangle_{L^{2}\left(\mathbb{S}^{1} \rightarrow \mathbb{R}\right)}
$$


where $D E(\Gamma)(\delta \Gamma)$ is the usual directional derivative of $E$ at $\Gamma$ along the direction $\delta \Gamma$. One of our goals for future work (discussed in the last section) is to extend our current framework to other inner products.

\subsection{Relating the two gradients}

Since $\phi$ is a function of $\Gamma$ (its SDF), and since $E(\Gamma)=F(\phi(\Gamma)) \forall \Gamma$, we have $D E(\Gamma)(\delta \Gamma)=D F(\phi)\left(\frac{d \phi}{d \Gamma}(\delta \Gamma)\right)$, i.e. $D E(\Gamma)(\delta \Gamma)=D F(\phi)(\delta \phi)$. On the one side:

$$
D E(\Gamma)(\delta \Gamma)=\left\langle\nabla_{\Gamma} E(\Gamma) \mid \delta \Gamma\right\rangle_{L^{2}\left(\mathbb{S}^{1} \rightarrow \mathbb{R}\right)}
$$

while on the other side, Eq.(5) and the definition of the gradient in Eq.(7) give :

$$
D F(\phi)(\delta \phi)=\left\langle\nabla_{\phi} F(\phi) \mid \delta \phi\right\rangle_{L^{2}(\Omega \rightarrow \mathbb{R})}=\left\langle\nabla_{\phi} F(\phi) \mid-\delta \Gamma\left(s_{x}\right) \cdot \boldsymbol{n}_{\Gamma\left(s_{x}\right)}\right\rangle_{L^{2}(\Omega \rightarrow \mathbb{R})}
$$

so that combining both sides gives:

$-\int_{\Omega} \nabla_{\phi} F(\phi)(x)\left(\delta \Gamma\left(s_{x}\right) \cdot \boldsymbol{n}_{\Gamma}\left(s_{x}\right)\right) d x=\int_{\Gamma} \nabla_{\Gamma} E(\Gamma)(s) \cdot \delta \Gamma(s) d \Gamma(s)$

We are now ready to derive a more explicit relation between these two gradients.

As we pointed out, the Lebesgue measure of the skeleton is 0 , and under smoothness assumptions about $F$, the integrand is bounded and consequently the integral over $\Omega$ is the same as the integral over $\Omega \backslash \operatorname{Skeleton}(\Gamma)$. We also note that any point $x \in \Omega \backslash \operatorname{Skeleton}(\Gamma)$ not on the skeleton can be written as:

$$
x=\Gamma\left(s_{x}\right)+\phi(x) \boldsymbol{n}_{\Gamma\left(s_{x}\right)}=\Gamma(s)+r \boldsymbol{n}_{\Gamma(s)}
$$

where $s(x)=s_{x}$ and $r(x)=\phi(x)$, which is illustrated in Fig. 3. Note that $r$ can be negative. We will define a new coordinate system using $s$ and $r$ such that the mapping that associates $x \in \Omega \backslash \operatorname{Skeleton}(\Gamma)$ with $(s, r)$ is injective. The infinitesimal (vector) elements of the two coordinate systems are related by:

$$
\begin{aligned}
d \mathbf{x} & =\left\|\frac{d \Gamma}{d s}\right\|_{\mathbb{R}^{2}} \boldsymbol{t}_{\Gamma(s)} d s+\boldsymbol{n}_{\Gamma(s)} d r-r \kappa_{\Gamma(s)}\left\|\frac{d \Gamma}{d s}\right\|_{\mathbb{R}^{2}} \boldsymbol{t}_{\Gamma(s)} d s \\
& =\left(1-\kappa_{\Gamma(s)} r\right)\left\|\frac{d \Gamma}{d s}\right\|_{\mathbb{R}^{2}} \boldsymbol{t}_{\Gamma(s)} d s+\boldsymbol{n}_{\Gamma(s)} d r
\end{aligned}
$$

where $\boldsymbol{t}_{\Gamma(s)}$ is the unit tangent of $\Gamma(s)$, and $\kappa_{\Gamma(s)}$ is the curvature of $\Gamma(s)$, which means by definition $\frac{d}{d s} \Gamma(s)=\left\|\frac{d \Gamma}{d s}\right\|_{\mathbb{R}^{2}} \boldsymbol{t}_{\Gamma(s)}$ and $\frac{d}{d s} \boldsymbol{n}_{\Gamma(s)}=-\kappa_{\Gamma(s)}\left\|\frac{d \Gamma}{d s}\right\|_{\mathbb{R}^{2}} \boldsymbol{t}_{\Gamma(s)}$. The determinant of the Jacobian $\left|\frac{d x}{d s}, \frac{d x}{d r}\right|$, which is the ratio between the infinitesimal area elements, is then $\left|1-\kappa_{\Gamma(s)} r\right|\left\|\frac{d \Gamma}{d s}\right\|_{\mathbb{R}^{2}}$. Therefore, the right side of Eq.(8) can be rewritten as:

$$
\begin{aligned}
-\int_{\Omega} \nabla_{\phi} & F(\phi)(x)\left(\delta \Gamma\left(s_{x}\right) \cdot \boldsymbol{n}_{\Gamma\left(s_{x}\right)}\right) d x \\
= & -\int_{\Omega} \nabla_{\phi} F(\phi)\left(x_{(s, r)}\right)\left(\delta \Gamma(s) \cdot \boldsymbol{n}_{\Gamma(s)}\right)\left|1-\kappa_{\Gamma(s)} r\right|\left\|\frac{d \Gamma}{d s}\right\|_{\mathbb{R}^{2}} d r d s \\
= & -\int_{\Gamma} \int_{l(s)} \nabla_{\phi} F(\phi)\left(x_{(s, r)}\right)\left|1-\kappa_{\Gamma(s)} r\right| d r \quad \boldsymbol{n}_{\Gamma(s)} \cdot \delta \Gamma(s) d \Gamma(s)
\end{aligned}
$$




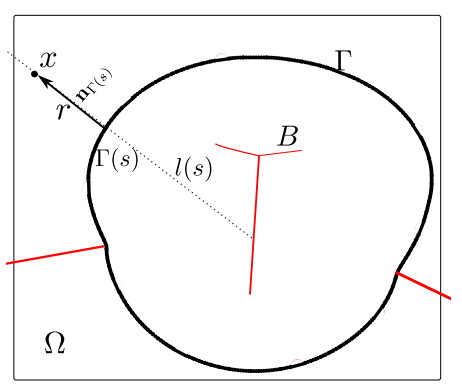

Fig. 3: Illustration of the change of coordinates. See text for explanations.

where $l(s)$ is the projection line that goes through $\Gamma(s)$ as illustrated in Fig. 3. It is the set of all points of $\Omega$ whose projection on $\Gamma$ is $\Gamma(s)$. It is thus a part of a line, which stops at the skeleton of $\Gamma$ and is also delimited by the boundary of $\Omega$. Therefore, a projection line $l(s)$ is a segment and the integral is well-defined. Since the equality Eq.(8) holds for all possible shape deformations $\delta \Gamma$, we obtain:

$$
\nabla_{\Gamma} E(\Gamma)(s)=-\int_{l(s)} \nabla_{\phi} F(\phi)\left(x_{(s, r)}\right)\left|1-\kappa_{\Gamma(s)} \phi\left(x_{(s, r)}\right)\right| d r \boldsymbol{n}_{\Gamma(s)}
$$

with $\phi\left(x_{(s, r)}\right)$ being just $r$ by definition. This is the key contribution of our work, since it draws the connection between the shape gradient $\nabla_{\Gamma} E(\Gamma)$ and the level set gradient $\nabla_{\phi} F(\phi)$ frequently used in the literature. The intuitive explanation of Eq.(9) is that the shape gradient $\nabla_{\Gamma} E(\Gamma)$ at $\Gamma(s)$ is a weighted integral of the level set gradient $\nabla_{\phi} F(\phi)$ along the projection line going through $\Gamma(s)$. We will shortly introduce a natural interpretation of these weights.

\subsection{The correct way to evolve the level sets}

Eq.(9) shows how to calculate the shape gradient $\nabla_{\Gamma} E(\Gamma)$ from the level set gradient $\nabla_{\phi} F(\phi)$. However, to actually update the level sets, we need to find the corresponding variation of $\phi$, that is $\delta \phi$. One possibility for this is to use the classical "velocity extension" approach $[9,10]$ where the velocity defined on $\Gamma$ is extended to $\Omega$. This involves the computation of the zero level set, which is sometimes undesirable. Another way is to directly express $\delta \phi$ using Eq.(5):

$$
\begin{aligned}
\delta \phi(x) & =-\delta \Gamma\left(s_{x}\right) \cdot \boldsymbol{n}_{\Gamma\left(s_{x}\right)} \\
& =-\int_{l\left(s_{x}\right)}\left|1-\kappa\left(s_{x}\right) \phi\left(x_{\left(s_{x}, r\right)}^{\prime}\right)\right| \nabla_{\phi} F(\phi)\left(x_{\left(s_{x}, r\right)}^{\prime}\right) d r
\end{aligned}
$$

To compute the variation $\delta \phi(x)$ at point $x$, it is thus sufficient to integrate the level set gradient, weighted by the area element ratio, along the projection line $l\left(s_{x}\right)$ that shares the same projection point as $x$. We will now give a natural geometrical interpretation of Eq.(9) and Eq.(10) which will lead to our numerical implementation algorithm. 


\subsection{Implementation}

Let us first examine the term $\left|1-r \kappa_{\Gamma(s)}\right|$ in Eq.(9). From basic differential geometry, we have that $\kappa_{\Gamma(s)}=\frac{d \theta}{d s}\left\|\frac{d \Gamma(s)}{d s}\right\|^{-1}=\frac{1}{R}$, where $R$ is the radius of the osculating circle at $\Gamma(s)$, with the same sign as the curvature, and where $d \theta$ is the angle formed by the normals to the curve at $\Gamma(s)$ and $\Gamma(s+d s)$, as illustrated in Fig. 4a. We also note that $r=\phi\left(x_{(s, r)}\right)$ and is negative when $x$ is inside $\Gamma$, and positive when $x$ is outside $\Gamma$. We can show that:

$\left|1-\phi(x) \kappa_{\Gamma(s)}\right| d \Gamma(s)=\left|1-\frac{r}{R}\right| d \Gamma(s)=\frac{|r-R|}{R} d \Gamma(s)=|r-R| d \theta=d L$

where $d L$ is the distance $x_{(s, r)}$ will travel for an infinitesimal step $d s$, i.e. the length of the arc formed by the projection lines through $\Gamma(s)$ and $\Gamma(s+d s)$, at a distance $|r-R|$ from their intersection, as shown in Fig. 4a. Note that $R<0$ in this figure. Since in Eq.(9) we are integrating a function of $x$ times $d L(r)$ along the projection line segment bounded on one side by the skeleton, we are interested in the region formed by the skeleton, the boundary of $\Omega$ and the projection lines going through $\Gamma(s)$ and $\Gamma(s+d s)$, that is, the red dotted region $d W$ shown in Fig. 4b. One can indeed show that, for any smooth function $f$ :

$$
\int_{d W(d s)} f(x) d x=\int_{r \in l(s)} f\left(x_{(s, r)}\right) d L(r) d r+o\left(\left\|\nabla_{x} f\right\|_{\infty} d s\right)
$$

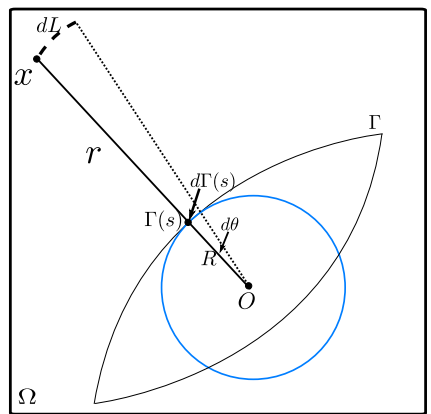

(a)

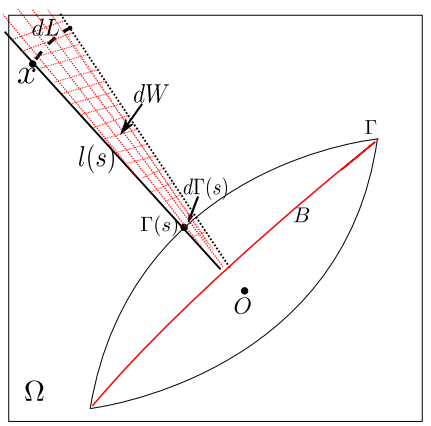

(b)

Fig. 4: Another look at velocity projection. (a) The osculating circle of $\Gamma(s)$ is shown in blue, where $O$ is the center of the osculating circle. (b) The skeleton of $\Gamma$ is $B$, shown in red. The area of the dashed subregion $d W$ is $\int_{r \in l_{s}} d L(r) d r=\int_{r \in l_{s}}\left|1-r \kappa_{\Gamma(s)}\right| d r d \Gamma(s)$.

The above analysis shows that Eq.(9) can be written as an integral over this subregion $d W$ when multiplied by an infinitesimal step $d \Gamma(s)$. We believe this is a more intuitive explanation of velocity projection, i.e. that the shape gradient 
at any point $\Gamma(s)$ is the average limit of the level set gradient over the region whose projection points are between $\Gamma(s)$ and $\Gamma(s+d s)$ :

$$
\nabla_{\Gamma} E(\Gamma)(s)=-\lim _{d s \rightarrow 0} \frac{1}{d \Gamma(s)} \int_{d W(d s)} \nabla_{\phi} F(\phi)(x) d x \boldsymbol{n}_{\Gamma(s)}
$$

Although both Eq.(5) and Eq.(12) are the same theoretically, we recommend using the integral over $d W$ for the following reason. To integrate along $l(s)$, we need to find the explicit range of $r$, whose estimation is not straightforward since it depends on the skeleton. We can however avoid the estimation of the skeleton if we integrate over regions $W$.

For any point $x$ in $\Omega$, we can easily locate its projection point $\Gamma\left(s_{x}\right)=$ $x-\phi(x) \nabla_{x} \phi(x)$. Suppose that $\Gamma$ is discretized by points $\Gamma\left(s_{i}\right), i=1, \ldots, N$, and there is a $W_{i}$ associated with each $\Gamma\left(s_{i}\right)$. Then Eq.(12) could be discretized as:

$$
\nabla_{\Gamma} E(\Gamma)\left(s_{i}\right)=-\sum_{x \in W_{i}} \nabla_{\phi} F(\phi)(x) \boldsymbol{n}_{\Gamma\left(s_{i}\right)}
$$

with its equivalent from Eq.(10) for direct level set evolutions, if $x \in W_{i}$ :

$$
\delta \phi(x)=\sum_{y \in W_{i}} \nabla_{\phi} F(\phi)(y)
$$

which means that the correct level set evolution can be computed from the level set gradient $\nabla_{\phi} F(\phi)$ very easily by just integrating over regions that share similar projection points.

However, since the projection $s_{x}$ of a random point $x \in \Omega$ is unlikely to be exactly one of the $s_{i}$, a point $x$ will typically contribute to more than one $W_{i}$. Let us denote by $h_{i}^{x}$ the weight that $x$ contributes to $W_{i}$. We have the constraint that all contributions of a point sum up to $1: \sum_{i} h_{i}^{x}=1$. Thus Eq.(13) is replaced by:

$$
\nabla_{\Gamma} E(\Gamma)\left(s_{i}\right)=-\sum_{x \in \Omega} h_{i}^{x} \nabla_{\phi} F(\phi)(x) \boldsymbol{n}_{\Gamma\left(s_{i}\right)}
$$

or, more practically, we obtain the level set evolution from Eq.(10):

$$
\delta \phi(x)=\sum_{i} h_{i}^{x} \sum_{y \in \Omega} h_{i}^{y} \nabla_{\phi} F(\phi)(y) .
$$

Then the problem comes down to how to estimate the weight $h_{i}^{x}$ that $x$ contributes to $W_{i}$ in a computationally effective manner. In practice, we assign $h_{i}^{x}$ to a function of the distance from $\Gamma\left(s_{x}\right)$ to $\Gamma\left(s_{i}\right)$ and normalize accordingly. A better numerical implementation algorithm is also one of our future goals.

\section{Implications for common level set gradients}

As mentioned earlier, much work has been carried out under the variational level set method, without considering whether the level set gradient agrees with the 
shape gradient. In this section, we will discuss some energy models that depend on SDFs and their gradients. We will not discuss energy models that depend only on $\Gamma$, such as the Geodesic Active Contour [16] and the Chan-Vese models [6], since our aim is to compare shape gradients with level set gradients.

We first consider the $L^{2}$ distance between two SDFs and its gradient:

$$
F(\phi)=\left\|\phi-\phi_{T}\right\|_{L^{2}}^{2}, \quad \nabla_{\phi} F(\phi)=2\left(\phi-\phi_{T}\right)
$$

Here $\phi_{T}$ is a target SDF. This energy is important and has many applications in shape analysis, morphing and shape prior image segmentation $[17,18]$. We are not aware of any work on computing the corresponding $E(\Gamma)$ or its shape gradient $\nabla_{\Gamma} E(\Gamma)$. Charpiat et al. [19] showed how to calculate the shape gradient directly with the $W^{1,2}$ norm but under a smooth approximation of infima. We can easily compute the shape gradient with our velocity projection step.

If instead, as in other works, we let $\phi$ evolve with the level set gradient, and rebuild it regularly from its 0-level to maintain its SDF property, we notice the following effect. Curve segments of $\Gamma$ that lie inside the region delimited by $\Gamma_{T}$ expand, while segments of $\Gamma$ that lie outside of $\Gamma_{T}$ shrink. This immediately implies that if $\Gamma$ lies completely outside of $\Gamma_{T}$, then the evolution process will shrink $\Gamma$ until it disappears no matter how close they are. This phenomenon is illustrated in Fig. 1a where we are trying to evolve the red circle $\Gamma$ to the black circle $\Gamma_{T}$. With the velocity projection approach, we can calculate the shape gradient and deform $\Gamma$ accordingly. Fig. 1b illustrates the deformation process under the correct shape gradient, which naturally morphs the initial curve to $\Gamma_{T}$.
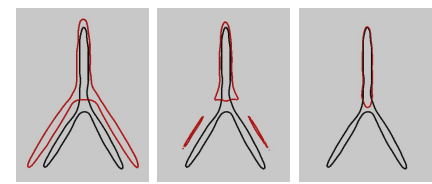

(a)
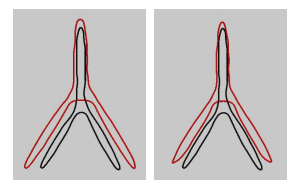

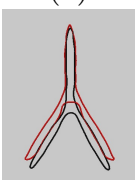

(b)
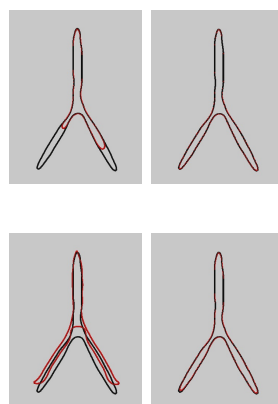

Fig. 5: Curve evolution of the red curve $\Gamma$, where the black curve $\Gamma_{T}$ is the target. The cost function is the $L^{2}$ distance between the two SDFs. (a) standard variational level set method, (b) our result.

Fig. 5 illustrates the evolution of two overlapped shapes using the same $L^{2}$ distance with and without velocity projection. Fig. 5a illustrates the traditional evolution without velocity projection. As we can see, the parts that are outside $\Gamma_{T}$ will shrink while the parts that are inside will expand. Fig. $5 \mathrm{~b}$ illustrates the 
correct deformation with velocity projection. As we can see, the deformation is more meaningful, leading to much better point correspondences. However, this model suffers from the drawback that the energy and thus the gradient depend on the domain $\Omega$. That is, by fixing $\Gamma$ and $\Gamma_{T}$ and changing $\Omega$ alone, we will get different energies, gradients and thus different deformation processes.

As a second example, consider the following energy model:

$$
F(\phi)=\int_{\Omega}\left(\phi-\phi_{T}\right)^{2} H(-\phi) d x
$$

which is the integration of the $L^{2}$ distance between $\phi$ and $\phi_{T}$ inside $\Gamma$. Here $H$ is the Heaviside function. This energy model was studied by Rousson and Paragios [7]. It can be shown that the level set gradient is:

$$
\nabla_{\phi} F(\phi)=2\left(\phi-\phi_{T}\right) H(-\phi)-\left(\phi-\phi_{T}\right)^{2} \delta(\phi)
$$

In this case, the velocity projection step is necessary to calculate the correct shape gradient. The evolution process of the correct shape gradient is illustrated in Fig. 6a. Since the integration is only inside $\Gamma$, it is not appropriate if $\Gamma$ lies outside $\Gamma_{T}$. To improve the evolution, we study the following symmetric term:

$$
F(\phi)=\int_{\Omega}\left(\phi-\phi_{T}\right)^{2} H\left(-\phi_{T}\right) d x
$$

which is the integration of the $L^{2}$ distance between $\phi$ and $\phi_{T}$ inside $\Gamma_{T}$. This energy was studied by Cremers and Soatto [8]. The level set gradient is:

$$
\nabla_{\phi} F(\phi)=2\left(\phi-\phi_{T}\right) H\left(-\phi_{T}\right)
$$

It is only defined within $\Gamma_{T}$ and therefore if we try to make $\Gamma$ evolve to $\Gamma_{T}$ under this gradient alone, most likely it won't move at all! We can again calculate its shape gradient (see Fig. 6b). This evolution does not correctly draw $\Gamma$ to $\Gamma_{T}$. The reason is that the level set gradient is non- 0 inside $\Gamma_{T}$ and all the projection points inside $\Gamma_{T}$ fall only on the blue curve segment of $\Gamma$ in Fig. 6c. Therefore, the fastest way to minimize this energy, or the gradient, is to only move the blue curve segment to $\Gamma_{T}$. The dissimilarity measure between $\Gamma$ and $\Gamma_{T}$ can be made symmetric by combining both Eq.(18) and Eq.(20):

$$
F(\phi)=\int_{\Omega}\left(\phi-\phi_{T}\right)^{2} H\left(-\phi_{T}\right) d x+\int_{\Omega}\left(\phi-\phi_{T}\right)^{2} H(-\phi) d x
$$

The evolution of this symmetric dissimilarity measure is shown in Fig. 6d. As we can see, it correctly evolves $\Gamma$ to $\Gamma_{T}$. We note here that the traditional variational gradient of this symmetric measure could also be used to evolve $\Gamma$. However, since the level set gradient Eq.(21) is not defined on curve segments of $\Gamma$ that lie outside $\Gamma_{T}$, only the gradient Eq.(19) would play a role in the evolution and it would not correctly draw $\Gamma$ to $\Gamma_{T}$. 


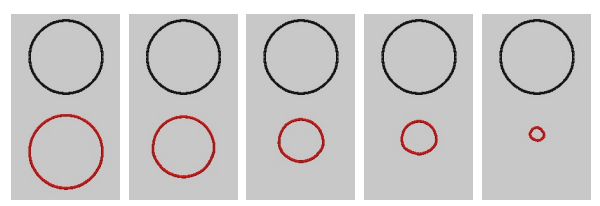

(a)
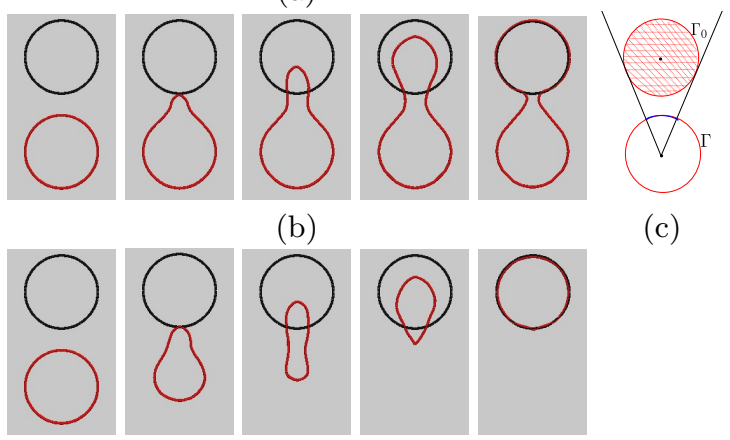

(c)

(d)

Fig. 6: The evolution from the red circle $\Gamma$ to the black circle $\Gamma_{T}$ under the correct shape gradient of the three different energy models. (a) energy model Eq.(18), (b) energy model Eq.(20), (c) the blue curve segment is the region that $H\left(-\phi_{T}\right)$ projects onto for (b), (d) energy model Eq.(22).

As a final example, we consider the following energy model [20]:

$$
E(\Gamma)=\int_{\Gamma_{T}} \phi^{2} d s=\int_{\Omega} \phi^{2} \delta\left(\phi_{T}\right) d x=F(\phi)
$$

This also defines a dissimilarity between $\Gamma$ and $\Gamma_{T}$. The level set gradient is:

$$
\nabla_{\phi} F(\phi)=2 \phi \delta\left(\phi_{T}\right)
$$

which is defined only along $\Gamma_{T}$ and is zero everywhere else. Therefore, it is also problematic for the traditional variational level set method. However, if we apply the velocity projection approach we can calculate the true deformation field:

$$
\delta \phi(x)=-2 \sum_{y \in\left(l\left(s_{x}\right) \cap \Gamma_{T}\right)}\left|1-\phi(y) \kappa\left(s_{x}\right)\right| \phi(y)
$$

where $l\left(s_{x}\right)$ is the projection line going through $x$. The deformation process is illustrated in Fig. 7a. However, as we can see, this evolution also does not correctly draw $\Gamma$ to $\Gamma_{T}$. The reason is the same as in Fig. 6c.

To improve the evolution process, we can add a second symmetric term:

$$
F(\phi)=\int_{\Gamma_{T}} \phi^{2} d s+\int_{\Gamma} \phi_{T}^{2} d s
$$

The evolution of this symmetric energy under the correct shape gradient is illustrated in Fig. 7c and it successfully draws $\Gamma$ to $\Gamma_{T}$. 


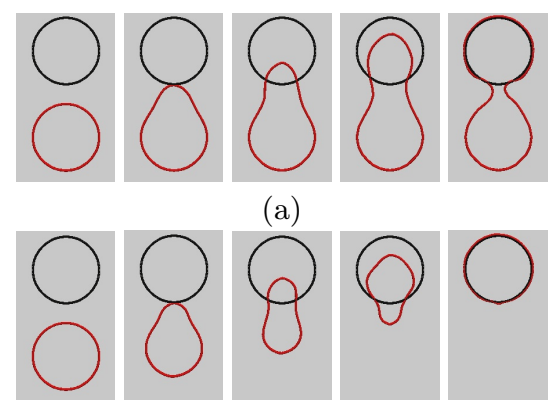

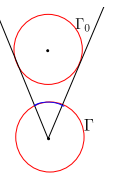

(b)

(c)

Fig. 7: The evolution from the red circle $\Gamma$ to the black circle $\Gamma_{T}$ under the shape gradient of two different energy models. (a) energy model Eq.(23), (b) the blue curve segment is the region that $\delta\left(\phi_{T}\right)$ projects onto, (c) energy model Eq.(26).

\section{Discussion and conclusions}

The experiments in Section 4 show that the limitations of traditional variational level set formulations can be fixed with our velocity projection step. In this work, we used shape morphing as a motivating application since it is closely related to other computer vision problems. In future work, we plan to apply our method to shape-prior image segmentation and statistical shape analysis. Fig. 5 shows the evolution of tubular structures under the traditional level set gradient can be problematic and our corrected shape gradient can handle these cases nicely.

We should point out that the geometric $L^{2}\left(\mathbb{S}^{1} \rightarrow \mathbb{R}\right)$ inner product has been shown to suffer from serious drawbacks as a metric on the manifold of shapes $[21,22]$. Specifically, the $L^{2}$ geodesic distance between two shapes is 0 . Since we consider gradient descents only, the $L^{2}$ inner product will pose no theoretical problem. However, we would like to investigate other inner products such as the $H^{1}\left(\mathbb{S}^{1} \rightarrow \mathbb{R}\right)$ inner product $[14,15]$. We are also investigating energy models other than $F(\phi)$, such as $F(\Gamma, \phi)$, as well as extending our framework to 3D.

\section{References}

1. Kass, M., Witkin, A., Terzopoulos, D.: Snakes: Active contour models. International journal of computer vision 1 (1988) 321-331

2. Osher, S., Sethian, J.: Fronts propagating with curvature-dependent speed- Algorithms based on Hamilton-Jacobi formulations. J. of Comp. Physics 79 (1988)

3. Osher, S., Fedkiw, R.: Level set methods and dynamic implicit surfaces. (2002)

4. Aubert, G., Barlaud, M., Faugeras, O., Jehan-Besson, S.: Image segmentation using active contours: Calculus of variations or shape gradients? SIAM Journal of Applied Mathematics 63 (2003)

5. Zhao, H., Chan, T., Merriman, B., Osher, S.: A Variational Level Set Approach to Multiphase Motion. Journal of Computational Physics 127 (1996) 179-195

6. Chan, T., Vese, L.: Active contours without edges. IEEE TIP 10 (2001) 266-277 
7. Rousson, M., Paragios, N.: Shape Priors for Level Set Representations. In: European Conference on Computer Vision. (2002)

8. Cremers, D., Soatto, S.: A pseudo-distance for shape priors in level set segmentation. In: IEEE workshop on variational, geometric and level set methods. (2003)

9. Gomes, J., Faugeras, O.: Reconciling distance functions and level sets. Journal of Visual Communication and Image Representation 11 (2000) 209-223

10. Adalsteinsson, D., Sethian, J.: The fast construction of extension velocities in level set methods. Journal of Computational Physics 148 (1999) 2-22

11. Solem, J., Overgaard, N.: A geometric formulation of gradient descent for variational problems with moving surfaces. In: Scale Space and PDE methods in computer vision. (2005)

12. Peng, D., Merriman, B., Osher, S., Zhao, H., Kang, M.: A PDE-Based Fast Local Level Set Method. Journal of Computational Physics 155 (1999) 410-438

13. Li, C., Xu, C., Gui, C., Fox, M.: Level set evolution without re-initialization: A new variational formulation. In: CVPR. (2005) 430-436

14. Charpiat, G., Maurel, P., Pons, J., Keriven, R., Faugeras, O.: Generalized gradients: Priors on minimization flows. IJCV 73 (2007) 325-344

15. Sundaramoorthi, G., Yezzi, A., Mennucci, A.: Sobolev active contours. International Journal of Computer Vision 73 (2007) 345-366

16. Caselles, V., Kimmel, R., Sapiro, G.: Geodesic active contours. IJCV 22 (1997)

17. Kim, J., Cetin, M., Willsky, A.: Nonparametric shape priors for active contourbased image segmentation. Signal Processing 87 (2007) 3021-3044

18. Cremers, D., Sochen, N., Schnorr, C.: Towards Recognition-Based Variational Segmentation Using Shape Priors and Dynamic Labeling. In: SSVM. (2003)

19. Charpiat, G., Faugeras, O., Keriven, R.: Approximations of shape metrics and application to shape warping and empirical shape statistics. Foundations of Computational Mathematics 5 (2005) 1-58

20. Gui, L., Thiran, J., Paragios, N.: Cooperative object segmentation and behavior inference in image sequences. IJCV 84 (2009) 146-162

21. Michor, P., Mumford, D.: Riemannian geometries on spaces of plane curves. Arxiv preprint math/0312384 (2003)

22. Yezzi, A., Mennucci, A.: Conformal metrics and true "gradient flows" for curves. In: ICCV. Volume 1. (2005)

\section{Appendix}

Proof of Eq.(5): $(\phi+\delta \phi)(x)=\phi_{\Gamma^{\prime}}(x)$ implies, using the signed distance $d$ :

$$
\delta \phi(x)=\phi_{\Gamma^{\prime}}(x)-\phi(x)=d\left(x, \Gamma^{\prime}\right)-d(x, \Gamma) .
$$

Since the part of an infinitesimal deformation that is tangent to $\Gamma$ has no effect on the shape (just reparameterizes), we only keep the part of the deformation that is normal to $\Gamma$, and the following redefinition of $\Gamma^{\prime}$ describes the same shape (as a set of points): $\quad \Gamma^{\prime}(s):=\Gamma(s)+\left(\delta \Gamma(s) \cdot \mathbf{n}_{\Gamma(s)}\right) \mathbf{n}_{\Gamma\left(s_{x}\right)}$.

$$
\text { Then } \begin{aligned}
x-\Gamma^{\prime}\left(s_{x}\right) & =x-\Gamma\left(s_{x}\right)-\left(\delta \Gamma\left(s_{x}\right) \cdot \mathbf{n}_{\Gamma\left(s_{x}\right)}\right) \mathbf{n}_{\Gamma\left(s_{x}\right)} \\
\left(x-\Gamma^{\prime}\left(s_{x}\right)\right) \cdot \mathbf{n}_{\Gamma\left(s_{x}\right)} & =\left(x-\Gamma\left(s_{x}\right)\right) \cdot \mathbf{n}_{\Gamma\left(s_{x}\right)}-\left(\delta \Gamma\left(s_{x}\right) \cdot \mathbf{n}_{\Gamma\left(s_{x}\right)}\right)\left(\mathbf{n}_{\Gamma\left(s_{x}\right)} \cdot \mathbf{n}_{\Gamma\left(s_{x}\right)}\right) \\
d\left(x, \Gamma^{\prime}\right) & =d(x, \Gamma)-\delta \Gamma\left(s_{x}\right) \cdot \mathbf{n}_{\Gamma\left(s_{x}\right)}
\end{aligned}
$$

since the projection of any point $x \in \Omega$ on the closed subset $\Gamma$ is necessarily orthogonal to its boundary $\Gamma$. Hence with Eq. $(27)$ one has $\delta \phi(x)=-\delta \Gamma\left(s_{x}\right) \cdot \mathbf{n}_{\Gamma\left(s_{x}\right)}$. 